\title{
GREAT/SOFIA atmospheric calibration *
}

\author{
X. Guan ${ }^{1}$, J. Stutzki ${ }^{1}$, U. U. Graf ${ }^{1}$, R. Güsten ${ }^{2}$, Y. Okada ${ }^{1}$, M. A. Requena-Torres ${ }^{2}$, \\ R. Simon ${ }^{1}$, and H. Wiesemeyer ${ }^{2}$ \\ 1 I. Physikalisches Institut, Universität zu Köln, Zülpicher Str. 77, 50937 Köln, Germany \\ e-mail: guan@ph1.uni-koeln.de \\ 2 Max-Plank-Institut für Radioastronomie, Auf dem Hügel 69, 53121 Bonn, Germany
}

Received 31 January 2012 / Accepted 27 February 2012

\section{ABSTRACT}

\begin{abstract}
The GREAT observations need frequency-selective calibration across the passband for the residual atmospheric opacity at flight altitude. At these altitudes the atmospheric opacity has both narrow and broad spectral features. To determine the atmospheric transmission at high spectral resolution, GREAT compares the observed atmospheric emission with atmospheric model predictions, and therefore depends on the validity of the atmospheric models. We discuss the problems identified in this comparison with respect to the observed data and the models, and describe the strategy used to calibrate the science data from GREAT/SOFIA during the first observing periods.
\end{abstract}

Key words. atmospheric effects - submillimeter: general

\section{Introduction}

The German REceiver for Astronomy at Terahertz frequencies $\left(\right.$ GREAT) ${ }^{1}$ (Heyminck et al. 2012) onboard the Stratospheric Observatory For Infrared Astronomy (SOFIA) (Becklin \& Gehrz 2009; Young et al. 2012) observes at typical flight altitudes of $8 \mathrm{~km}$ (where telescope- and instrument set-up starts) up to $14 \mathrm{~km}$. The atmospheric transparency at these altitudes is high (though not unity) except near strong absorption lines of species such as water and other trace constituents $\left(\mathrm{O}_{3}, \mathrm{CO}, \mathrm{N}_{2} \mathrm{O}\right.$, etc.). The typical amount of water vapor in the atmosphere above the observatory (precipitable water vapor, $p w v$ ) is in the range of a few $\mu \mathrm{m}$ at very good observing conditions and up to $50 \mu \mathrm{m}$ and higher at bad conditions. The atmospheric pressure, rapidly decreasing with increasing altitude, results in narrower absorption lines with increasing altitude. As a result, the atmospheric opacity at flight altitude shows many narrow spectral features, typically with a width of about $100 \mathrm{MHz}$ down to a few $\mathrm{MHz}$ (varying with species and altitude at which the particular species absorbs). In addition, extended line wings from very strong absorption lines, in particular of water, and collision-induced absorption (CIA), where the collisions of the absorbing species with other molecules generate a temporary dipole moment, contribute to a spectrally smooth broad-band opacity.

The correction of airborne astronomical data for the atmospheric transmission is thus much more challenging than for ground-based observations, because of the small-scale variations resulting from the narrow atmospheric lines. Direct

\footnotetext{
* Appendix A is available in electronic form at http://www. aanda.org

${ }^{1}$ GREAT is a development by the MPI für Radioastronomie (Principal Investigator: R. Güsten) and KOSMA/Universität zu Köln, in cooperation with the MPI für Sonnensystemforschung and the DLR Institut für Planetenforschung.
}

measurement of the frequency-dependent transmission is in principle possible, but is limited by the inherently higher noise in narrow frequency-bins. Hence, the calibration has to rely on appropriate models of the frequency-dependent atmospheric opacity and the proper approach is to determine the transmission from model fits to the observed sky emission. This approach has been implemented and successfully used in recent years at ground-based high-altitude observatories such as APEX (Güsten et al. 2006) and NANTEN2 (Kawamura et al. 2005) in Chile. The proper description of the frequency-dependent atmospheric transmission is particularly important for absorption measurements against continuum background sources, because the background emission is also modulated by the atmospheric transmission, which can therefore interfere with the absorption profiles from the astronomical sources.

We first discuss the calibration approach in Sect. 2 and its implementation for GREAT on SOFIA in Sect. 3. GREAT allows us for the first time to simultaneously observe two widely separated frequency bands in the THz-regime at high spectral resolution. Accordingly, it has come as no surprise that the atmospheric models currently in use for predicting the THz-regime emission and absorption of the atmosphere turn out to have clear deficiencies in properly modeling the atmosphere above SOFIA to the degree necessary to consistently calibrate the GREAT data. These problems are discussed in Sect. 4. We conclude by describing the pragmatic approach chosen for the calibration of the GREAT data of the first observing season and the perspectives for future improvements in Sect. 5.

\section{Approach to atmospheric calibration}

Following the notation of Appendix A, the sky brightness on an equivalent antenna temperature scale along a line-of-sight at 
elevation $E l$ for a plane parallel atmosphere, i.e. taking for the airmass the approximation $A=1 / \sin (E l)$, is given by

$$
\begin{aligned}
T_{\mathrm{A}, \mathrm{sky}, v} & =\int_{0}^{\tau_{Z, v}} \mathcal{J}_{v}\left(T\left(\tau_{v}^{\prime}\right)\right) \times \mathrm{e}^{-\tau_{v}^{\prime} A} \mathrm{~d}\left(\tau_{v}^{\prime} A\right) \\
& =: \tilde{T}_{\mathrm{sky}, v}\left(1-t_{\mathrm{a}, v}\right),
\end{aligned}
$$

with the atmospheric transmission

$t_{\mathrm{a}, v}=\exp \left(-\tau_{Z, v} / \sin (E l)\right)$,

the frequency-dependent zenith opacity $\tau_{Z, v}$, and the definition of the effective sky brightness

$\tilde{T}_{\mathrm{sky}, v}=T_{\mathrm{A}, \mathrm{sky}, v} /\left(1-t_{\mathrm{a}, v}\right)$

according to Eq. (A.7).

An often used approximation is that the emission occurs at the ambient temperature of the atmosphere near the observatory, $T_{\mathrm{amb}}$, so that

$\tilde{T}_{\mathrm{sky}, v} \approx \mathcal{J}_{v}\left(T_{\mathrm{amb}}\right)$,

although this assumption may not be particularly well justified for absorption lines from the higher atmosphere, which is warmer than at flight altitude (see Sect. 3).

In principle, the above equation can be inverted to calculate the sky transmission at each frequency from the measured sky brightness. However, because the atmospheric calibration has to be frequency specific, deriving the opacity from the skyemission in each frequency bin results in quite large errors due to the limited signal-to-noise ratio $(\mathrm{S} / \mathrm{N})$ in their narrow bandwidth. In addition, a heterodyne instrument receiving double sideband (DSB), such as GREAT, folds the signal- and imagesideband on top of each other, so that the emission from the two sidebands cannot be separated. To overcome these problems, one has to (i) use an atmospheric model to predict the atmospheric opacity as a function of frequency at the given observing altitude and line-of-sight, parameterized by a few dominant atmospheric parameters, such as the pwv and the ambient temperature, (ii) derive these parameters from the comparison between the observed emission and the model, and (iii) then use the model opacities, calculated with these best-matching parameters, to correct for the attenuation of the astronomical signal.

This can be done either for individual spectrometer channels to only de-convolve the image- and signal-sideband opacity, or, to increase the $\mathrm{S} / \mathrm{N}$, over selected sub-bands or the complete reception bandwidth of the receiver (which in the case of GREAT might include the two simultaneously observed THz-bands $L 1$ and $L 2$ ). A necessary condition for the consistency of the calibration approach is that the atmospheric parameter(s) derived for individual channels or sub-bands are identical to within the noise and/or that the residual of the comparison between the atmospheric model and the observed sky-brightness is within the observed noise level. If this is not met, the atmospheric model obviously does not describe the actual atmosphere satisfactorily (or the measurements are corrupt).

The zenith opacity above the observer at altitude $h$ depends on the altitude profile of the abundances of the atmospheric species that are responsible for the absorption, which is driven by the complex chemical processes in the atmosphere, as well as by the pressure profile (influencing the pressure broadening) and temperature profile (determining the population of the relevant species within the absorbing states). All these may vary as a function of geographical position and local weather parameters. To arrive at a manageable problem, one has to make additional assumptions, such as that the altitude variations of abundances, temperatures, and pressure are given by a standard atmosphere, e.g., the US Standard Atmosphere (1976), which specifies the altitude variation on a pressure altitude vertical scale (in the following, altitude often refers to pressure altitude). Similarly, it specifies the total abundances and abundance variation with altitude of the atmospheric species of interest, except for a few, where their distribution has to be adapted to the specific observing conditions, as discussed below. In particular the column of water molecules above the observer, pwv, may change rapidly, because it is largely determined by local weather. Hence, a useful parameterization of the atmospheric absorption is given by splitting the wet, i.e., water absorption, and the dry part as

$$
\begin{aligned}
\tau_{0, v}= & b_{v}\left(p_{\mathrm{amb}}\left[, T_{\mathrm{amb}}, X_{j}, \ldots\right]\right) \times p w v \\
& +c_{v}\left(p_{\mathrm{amb}}\left[, T_{\mathrm{amb}}, X_{j}, \ldots\right]\right),
\end{aligned}
$$

where the wet and dry coefficients are a function of the ambient pressure $p_{\text {amb }}$ (or altitude), and additional atmospheric parameters such as the ambient temperature $T_{\mathrm{amb}}$, abundances $X_{j}$, and possibly other parameters that, e.g., specify the altitude profile of these abundances. A particular atmospheric model can then be represented by a multi-dimensional look-up-table of the $b$-, $c$-coefficients vs. frequency, (pressure) altitude, and additional parameters, which are derived from the various atmospheric models by fitting the model opacities, calculated at two values of $p w v$ and at different frequencies and altitudes. The details on deriving the $b$ - and $c$-coefficients are given in Appendix A.

\subsection{Atmospheric models}

Several atmospheric models are in use within the radio- and FIRastronomy community, both for ground-based observatories and air-borne applications. For the GREAT/SOFIA calibration we have investigated the use of AM (Paine 2011), ATRAN (Lord 1992), and MOLIERE (Urban et al. 2004). The ATM-model (Pardo et al. 2001), in use for ALMA and other ground-based observatories, in its publically available form does not include the higher excitation water lines and hence is of limited use for frequencies above $1.5 \mathrm{THz}$.

Figure 1, comparing the wet- and dry-atmospheric coefficients of the three models, shows that ATRAN at present does not include the CIA from $\mathrm{N}_{2}$ and $\mathrm{O}_{2}$, which is responsible for the quasi-continuum opacity shown in the other two models. The atmospheric opacity due to these CIA-processes differs by a factor of $\approx 2$ between MOLIERE and AM, being higher for MOLIERE. All three models agree on the wet atmospheric opacity, although ATRAN has some small errors in the frequencies of a few higher excitation water lines. Because ATRAN does not include the dry-atmosphere continuum opacity, and the dry-atmosphere contribution to the opacity due to CIA-processes is already too high compared to the observed sky emission (see below) even for the AM model, we used the AM model for the standard GREAT calibration.

To calculate the atmospheric transmission for different pwvvalues, we modeled the water mixing ratio as an exponential function with altitude in the high troposphere and low stratosphere, and with a constant, but low value higher above, following Mastenbrook (1968) and Herbin et al. (2009). The detailed treatment is presented in Appendix A.

The nitrous oxide, $\mathrm{N}_{2} \mathrm{O}$, profile is known from ground- and balloon observations (Emmons et al. 1994; Camy-Peyret et al. 2004; Strong et al. 2008). We adapted a simplified profile for use with $\mathrm{AM}$. It uses a $\mathrm{N}_{2} \mathrm{O}$ mixing ratio of $0.3 \mathrm{ppm}$ below an 


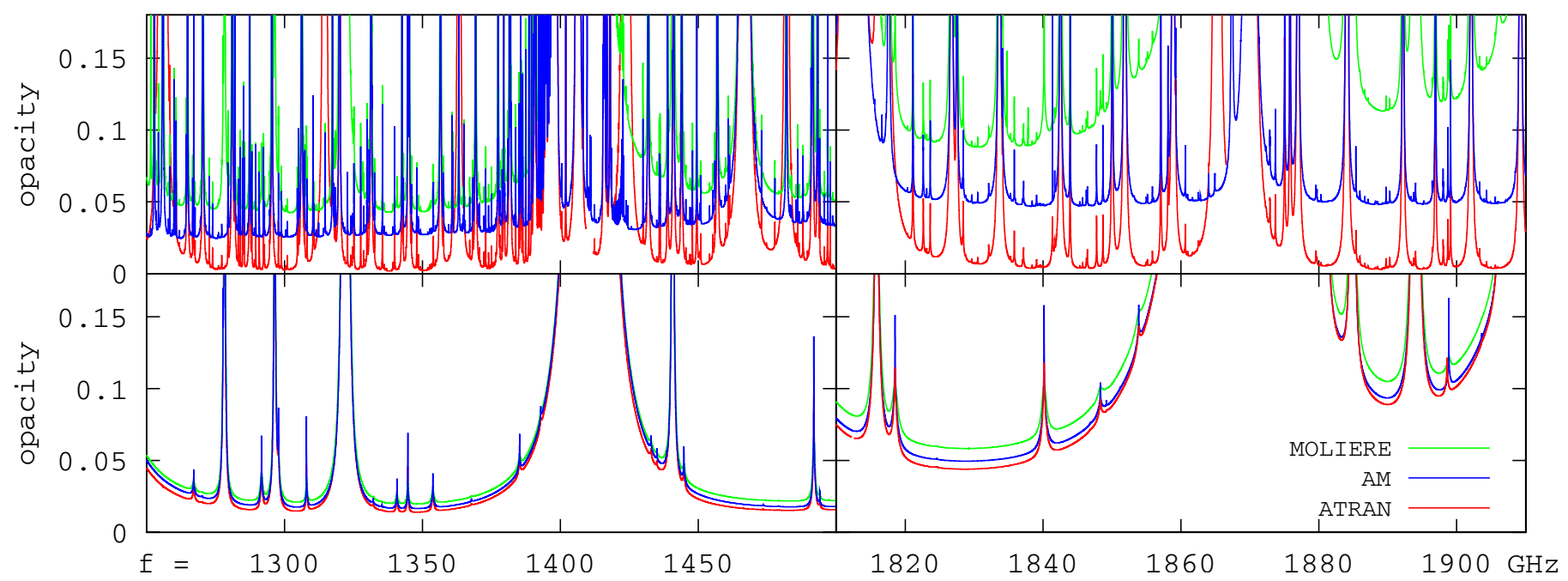

Fig. 1. Comparison of the dry (top) and wet (bottom) opacity coefficients as defined in Eqs. (6), (A.29) and (A.27) for the frequency range of GREAT channels $L 1$ (left) and L2 (right), calculated from the atmospheric models AM, ATRAN and MOLIERE (see text), showing the opacity for a pressure altitude of $p_{\mathrm{amb}}=133$ Torr and a $p w v=10 \mu \mathrm{m}$. The largest discrepancy is in the quasi-continuous dry-atmospheric opacity due to collision-induced absorption (CIA), which is completely neglected in ATRAN and differs by about a factor of 2 between MOLIERE and AM.

altitude of $60 \mathrm{hPa}$, and $0.1 \mathrm{ppm}$ above. This is a rough approximation of many measured profiles, such as that given in Fig. 1 of Camy-Peyret et al. (2004). $\mathrm{N}_{2} \mathrm{O}$ does not produce particularly strong atmospheric features, but we adapted an $\mathrm{N}_{2} \mathrm{O}$ profile based on real observations, to compare AM with ATRAN, which produces far too strong $\mathrm{N}_{2} \mathrm{O}$ features compared with the observed sky emission.

The ozone, $\mathrm{O}_{3}$, profile changes rapidly with geographic positions and with season. The profile actually used in the GREAT calibration is obtained by fitting sky measurements with the NANTEN2 telescope in Chile to properly reproduce the strength of the ozone lines. The GREAT/SOFIA calibration shows that the measured ozone lines are adequately reproduced by the model, so that this altitude profile is also applicable here (although a few SOFIA measurements show an increased ozone abundance compared to this model, which does not affect the overall calibration, because the ozone lines are very narrow spectral features, but has to be taken into account for absorption measurements against a continuum source).

\section{Implementation for GREAT}

The calibration of atmospheric transmission for GREAT/SOFIA is implemented in the task kalibrate as part of the kosma_software observing software package. The same scheme is used for the submm-wave data at the NANTEN2 observatory in Chile. It fits the observed, calibrated differential sky-hot spectrum with the atmospheric model brightness as given by Eqs. (A.48) and (A.47) (and uses the approximation $\omega=\Omega=0$ and $\tilde{T}_{\text {sky }, v}=\mathcal{J}_{v}\left(T_{\text {sky }}\right)$ as detailed in Appendix A). The best-fit value is thus determined with high precision, making use of the full spectral information observed, i.e., with a good S/N. The user can control whether the fit is performed as a "common" fit to all simultaneously observed spectra (several receiver pixels at several frequency bands) or individually for each spectrometer. The atmospheric transmission for this best-fit-value is then applied to the full spectra channel by channel, thus keeping the full frequency resolution of the atmospheric features (to the resolution with which the frequencies where sampled when generating the $b$-, $c$-coefficient look-up tables).
The approximation $\tilde{T}_{\text {sky }, v}=\mathcal{J}_{v}\left(T_{\text {sky }}\right)$ assumes that all opacity originates at the same temperature. To check this assumption, Fig. 2 shows the effective sky brightness $\tilde{T}_{\mathrm{sky}, v}$ (Eq. (4)), calculated with the AM model from the model brightness and opacity for a frequency range near a strong water line at two different $p w v$-values. The dry-atmospheric lines (shown in the middle panel in Fig. 2), as well as the narrow core of the high excitation water line (see lower panel in Fig. 2), originate in the higher stratosphere, where the temperature increases well above the ambient temperature at the observer's altitude in the lower stratosphere. At the low pwv of $3 \mu \mathrm{m}$, the effective sky brightness temperature in these lines is therefore substantially higher than the Rayleigh-Jeans-(R-J) corrected ambient sky temperature of about $172 \mathrm{~K}$. With higher pwv, the increasing opacity at altitudes, and hence temperatures, near the observer's altitude absorbs these emission lines from the high atmosphere, so that the excess effective sky temperature near these lines is reduced and the effective sky temperature approaches the ambient temperature. The excess is typically up to about $10 \mathrm{~K}$ at very low $p w v$. Neglecting it results in a slight underestimation of the sky brightness in the cores of these lines for the model fit to the observed brightness. Because this affects only narrow frequency ranges, it has a negligible effect on the derived fit parameters.

\section{Application to GREAT data}

This calibration scheme, when applied to the GREAT data, is not able to self-consistently fit the atmospheric emission simultaneously for the $L 1$ and $L 2$ bands, whereas independent fits to the individual receiver bands converge well, but on solutions with different values of $p w v$ for each band. Comparison of the fits with the observed sky-hot spectra shows that the narrow spectral features resulting from the dry atmosphere and the line features of water are reproduced well. This indicates that the problem lies in the quasi-continuum opacity of the dry atmosphere, i.e., the approximately constant offset of the $c$-coefficients visible in Fig. 1 (resulting largely from the CIA-processes of $\mathrm{N}_{2}$ and $\mathrm{O}_{2}$ ) predicted in the models.

A closer look confirms this and shows that this quasicontinuum opacity of the dry atmosphere is too high. Indeed, independent fits to the emission in the two receiver bands for 


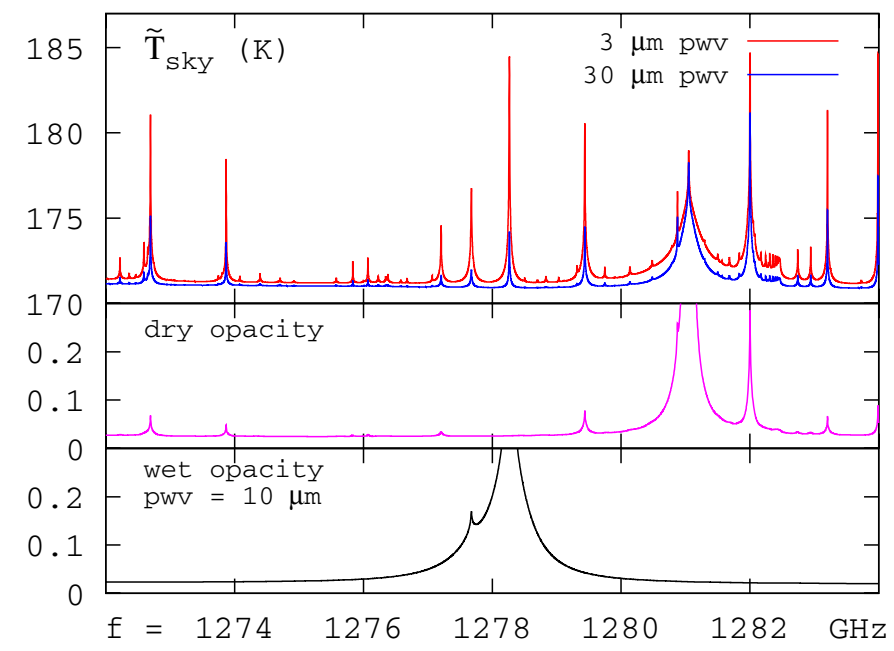

Fig. 2. Effective sky brightness, $\tilde{T}_{\text {sky }}$, (top panel) in a representative frequency window near a water line and several dry-atmospheric lines originating in the higher, warmer atmosphere, calculated with the AM model at a pressure altitude of 133 Torr and an ambient temperature of $200 \mathrm{~K}$, for two values of $p w v, 3$ (red) and $30 \mu \mathrm{m}$ (blue). The lower two panels show the dry atmospheric opacity (i.e., the $c$-coefficient; middle) and the wet atmospheric opacity at $10 \mu \mathrm{m} p w v$, i.e., $b \times 10 \mu \mathrm{m}$; bottom), similar to Fig. 1 to identify the spectral features causing the excess $e f$ fective brightness temperature.

observations with very good transmission converge to a formal fit result with slightly negative $p w v$-values: because the model opacity cannot be reduced below the dry-atmospheric contribution, i.e., the (too high) value of the $c$-coefficient, this excess opacity has to be compensated by negative $p w v$, and hence a negative wet opacity, $b \times p w v$, to match the observed low brightness of the sky at these low opacities (because negative values for $p w v$ are unphysical, the fit result is reset to $p w v=0$ in these cases).

Observations at higher overall opacity, i.e., low elevation and higher $p w v$, show a similar discrepancy. Figure 3 shows such an example for a tuning that observes the $\mathrm{CO}(11-10)$ line in $L 1$ and the CO (16-15) line in L2. Separate fits (black lines in Fig. 3) give $p w v=12.3 \mu \mathrm{m}$ for the $L 1$ band and $35 \mu \mathrm{m}$ for the $L 2$ band. The excess opacity in the model needs to be compensated in the fitting by a more reduced wet opacity, hence reduced $p w v$ values, than would be needed with the proper dry opacity. The scaling factor between the wet opacity and $p w v$, namely the $b$-coefficient as defined above, is typically higher by a factor of 2 to 3 for the $L 2$ band, so that the excess dry opacities in the model result in accordingly scaled, i.e., smaller differences in $p w v$ for the fitted pwv-value for the $L 2$ band compared to $L 1$.

Similarly, a common fit to both bands, now forced to using a unique value for $p w v$, results in a $p w v$-value somewhere in between the two discrepant values fitted in the separate fits. It overestimates the opacity in the band with lower opacity and underestimates the opacity in the other, higher opacity, band as shown by the green and red fit line in Fig. 3, which corresponds to a best-fit value of $21.4 \mu \mathrm{m} p w v$.

The above holds for tunings where the wet atmosphere does not contribute any line feature, but merely a continuum opacity (with a slight slope). In the rare cases where the wet atmosphere also contributes with a line feature, even the single frequency band fits may not converge to a consistent result: the line intensity forces a certain amount of water and hence also continuous opacity, so that the total opacity, including the dry opacity

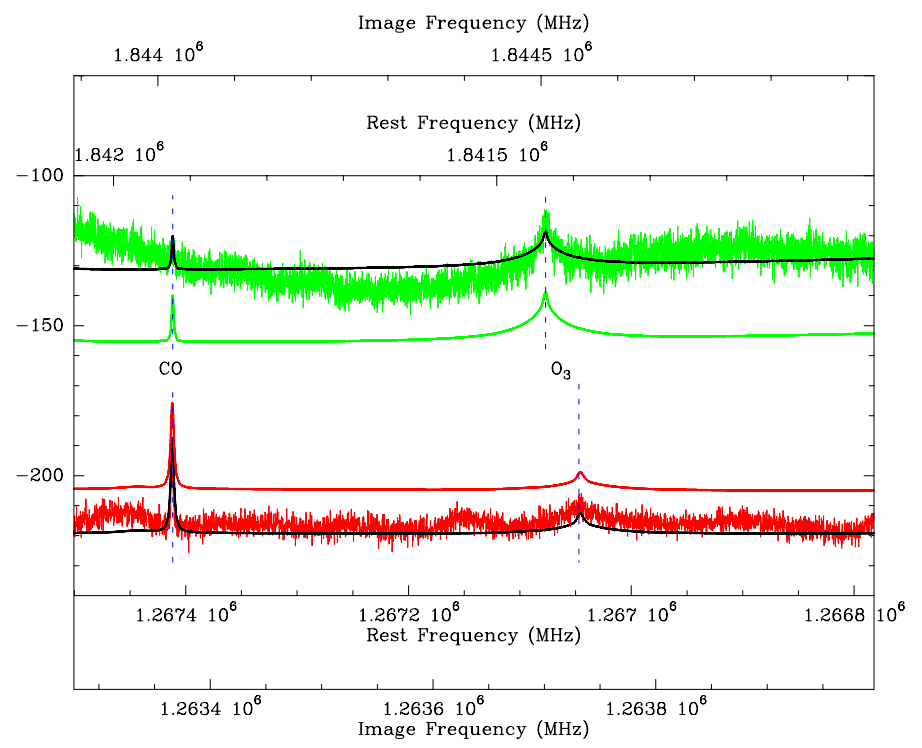

Fig. 3. Example $T_{\text {sky }}-T_{\text {hot }}$ spectra from simultaneous observations in the $L 1$ (red) and $L 2$ (green) band, showing two ozone lines (the broad features toward the middle of the $L 1$ and $L 2$ passband) and two CO lines (the narrow peaks on the left). Note that the two CO lines appear at the same velocity on a $v_{\mathrm{LSR}}$ scale, because the receiver was tuned to two $\mathrm{CO}$ lines for the astronomical observations. The two black curves are independent fits of $T_{\text {sky }}-T_{\text {hot }}$ for the two bands. The red and green lines between the measured spectra are the result of a common fit, which simultaneously fits a single value of pwv to both bands. In a common fit, a consistent value of $p w v$ cannot be derived for both bands.

overestimated by the atmospheric model, overshoots the range that is consistent with the observed atmospheric emission.

To confirm these findings, we modified the fitting procedure to include additional fit parameters $o_{L 1}$ and $o_{L 2}$, namely a frequency dependent dry-offset, so that Eq. (6) changes to

$\tau_{v}=b_{v} p w v+c_{v}- \begin{cases}o_{L 1}, & v \text { in } L 1 \\ o_{L 2}, & v \text { in } L 2 .\end{cases}$

This gives a more consistent fit, but not with unique offsets $o_{v}$ for different tunings and an ambiguous separation into pwv offsets and values for $o_{v}$. Similarly, one could use an empirical atmospheric model such as a linear combination of two of the models above. In practice the two extra parameters of course give a better fit to the data, but allow different combinations of their values and thus do not constrain the fit toward a better insight into the atmospheric model deficiency.

In principle, one can estimate the value by which the modelpredicted quasi-continuum dry opacity is too high compared to the observations by analyzing the data taken at very low $p w v$, i.e., where the formal fit gives negative values for $p w v$. However, this requires a very precise measurement of the low residual skybrightness at these low opacities. Despite the excellent stability of GREAT, documented by the Allan-variance minimum times of around $100 \mathrm{~s}$ (Heyminck et al. 2012), slight drifts in the total power gain profile caused by, e.g., mechanical deformation under gravity of the diplexer optics or residual drifts with temperature in the receiver electronics, limit the precision with which these measurements can be made. Figure 4 shows that these gain variations on time-scales of several minutes, i.e., between subsequent hot-cold gain-calibration scans, are on the order of up to a few percent. These relative errors in the gain translate into absolute errors in the derived opacities, because the corresponding sky-hot-observations are affected at the same level. Note that 


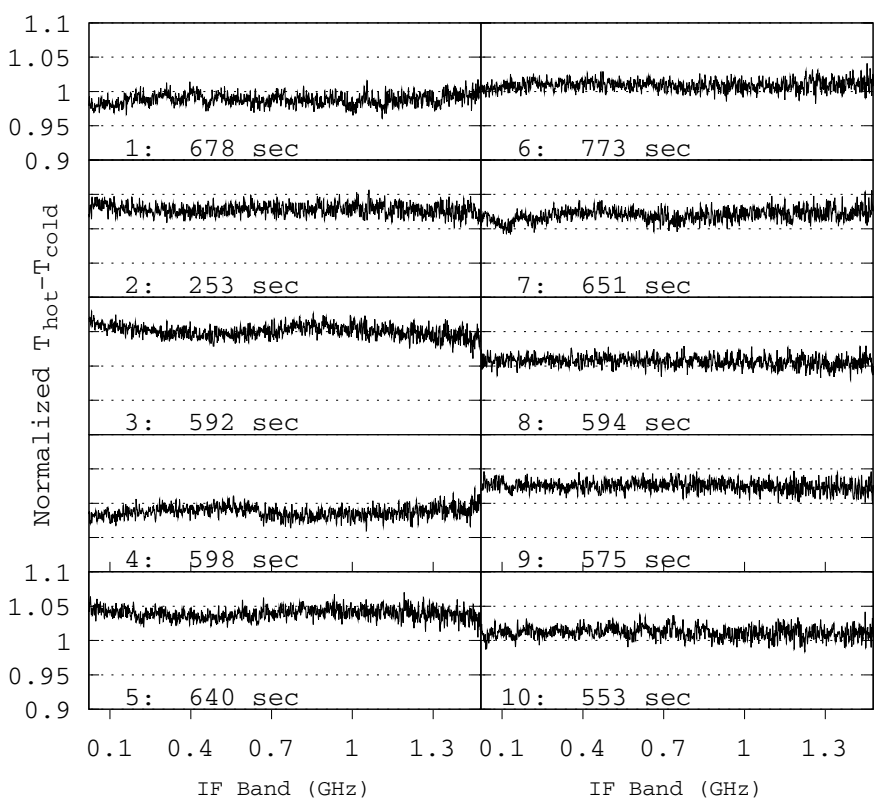

Fig. 4. Time series of hot-cold load-calibration scans (in the GREAT L2 channel), displayed across the central part of the IF band of width $1.4 \mathrm{GHz}$, calibrated against the previous load scan and normalized to the R-J-corrected hot-cold brightness difference. It shows that the receiver gain drifts by up to a few percent over time scales of several minutes. The sequence number and time offset to the previous scan is given in the bottom left corner of each panel.

absolute errors in the opacity of this order are perfectly tolerable for the calibration of the atmospheric transmission, because they contribute a small relative error of similar magnitude to the transmission, and hence to the intensity of the calibrated spectrum. But they compromise a more precise analysis of the atmospheric model discrepancies discussed above, because we are talking about small offsets, i.e., corrections to the $c$-coefficient on the order of a few times 0.01 . Only the few sky-measurements immediately following a load-measurement avoid this problem, but there are too few at very low opacity within the data set from the SOFIA basic-science flights, and these are scattered over different receiver tunings, so that they do not give sufficient statistics to derive a consistent estimate of the model discrepancy.

\section{Summary and outlook}

Given the problems in the atmospheric models as discussed above, the GREAT data from the basic science series of flights were calibrated using individual fits to each receiver band. Although these give inconsistent values for the best-fit $p w v$ for simultaneous observations, the relevant entity entering the calibration of the data is the transmission and not pwv. The calibration thus does not take advantage of the higher signal-to-noise that would be achievable with a common fit to the full reception bandwidth of the receiver, but it is nevertheless sufficiently precise to not introduce any significant errors (depending on the detailed circumstances of the observing conditions, we estimate the error in the atmospheric transmission thus derived to be on the order of a few up to a maximum of about 10\%). Correcting for the atmospheric transmission by applying the frequencydependent opacity as given by the atmospheric model, including the narrow spectral features resulting from the dry atmosphereat full spectral resolution, is crucial, however, to remove residuals from these features in the resulting astronomical spectrum, in particular for absorption measurements against a continuum source.

To improve the calibration, the inconsistencies in the atmospheric models with regard to the level of broad-band, quasicontinuum opacity that we identify with the GREAT/SOFIA observations in the THz-regime have to be overcome by developing a better understanding of the broad-band, quasi-continuous opacity contributed by the CIA-processes; in particular, its broad-band variation across the frequency range covered by the different channels of GREAT needs to be properly understood. In parallel, a careful analysis of the sky-measurements available from the basic-science flight series, but also dedicated future observations tuned to particular frequency settings with strong, but narrow water lines from the slightly higher atmosphere that may allow us to independently fix the pwv value and thus to resolve the ambiguity between dry continuous and wet continuous opacity contributions, will help to quantify and possibly correct the discrepancy in the models.

Acknowledgements. We thank the SOFIA engineering and operations teams, whose tireless support and good-spirit teamwork has been essential for the GREAT accomplishments during Early Science, and say Herzlichen Dank to the DSI telescope engineering team. SOFIA Science Mission Operations are conducted jointly by the Universities Space Research Association, Inc., under NASA contract NAS2-97001, and the Deutsches SOFIA Institut under DLR contract 50 OK 0901. IRAM (Grenoble, France) has implemented the GREAT/SOFIA specific CLASS header extensions, that allow to store the air-borne specific calibration parameters within the observation headers. Thanks go to Scott Paine, Steve Lord, Nicola Schneider and Juan Pardo for help with implementing and using the atmospheric models AM, ATRAN, MOLIERE and ATM. The development of GREAT was financed by the participating institutes, the Max-Planck-Society and the Deutsche Forschungsgemeinschaft within the framework of the SFBs 494 and 956.

\section{References}

Bauer, A., Godon, M., Kheddar, M., et al. 1987, JQSRT, 37, 531

Bauer, A., Godon, M., Kheddar, M., et al. 1989, JQSRT, 41, 49

Becklin, E. E., \& Gehrz, R. D. 2009, ASPC, 417, 101

Camy-Peyret, C., Payan, S., Dufour, G., et al. 2004, ESA SP, 562, 31

Downes, D. 1989, LNP, 333, 351

Emmons, L. K., Reeves, J. M., Shindell, D. T., et al. 1994, OZTS, 543

Güsten, R., Nyman, L. Å., Schilke, P., et al. 2006, A\&A, 454, 13

Herbin, H., Hurtmans, D., \& Clerbaux, C. 2009, ACP, 9, 9433

Heyminck, S., Graf, U. U., Güsten, R., et al. 2012, A\&A, 542, L1

Kawamura, A., Mizuno, N., Yonekura, Y., et al. 2005, IAUS, 235, P275

Kutner, M. L., \& Ulich, B. L. 1981, ApJ, 250, 341

Lord, S. 1992, NASA-TM-103957

Mastenbrook, H. J. 1968, JAtS, 25, 299

Paine, S. 2011, SMA memo, 152

Pardo, J. R., Cernicharo, J., \& Serabyn, E. 2001, IEEE Trans. Antennas Prop., 49/12, 1683

Strong, K., Wolff, M. A., Kerzenmacher, T. E., et al. 2008, ACP, 8, 4759

Urban, J., Baron, P., \& Lautié, N. 2004, JQSRT, 83, 529

US Standard Atmosphere 1976, NASA-TM-X-74335

Young, E. T., Becklin, E. E., Marcum, P. M., et al. 2012, ApJ, 749, L17 


\section{Appendix A: Atmospheric calibration of heterodyne astronomical observations}

This appendix is a complete compilation of the calibration scheme for astronomical observations with a heterodyne receiver, taking into account the special problems that appear at highest frequencies, i.e., at the edge or beyond the validity of the common RayleighJeans (R-J) approximation, where the R-J correction across the receiver input band has to be taken into account with the high IF-bandwidths in use at present instruments. It also describes a general scheme for incorporating the correction for atmospheric transmission, which for observations at high altitudes from the ground, but in particular from air-borne or balloon-borne altitudes requires a detailed treatment of the spectrally narrow atmospheric features.

The terminology follows, to the extent possible, the notation defined in Kutner \& Ulich (1981) and Downes (1989).

\section{A.1. Specific intensity and equivalent brightness temperature}

The specific intensity $I_{v}$ is expressed as an antenna brightness temperature $T_{\mathrm{A}, v}$ in the usual radio-astronomical notation,

$T_{\mathrm{A}, v}=\frac{\lambda^{2}}{2 k} I_{\nu}$

For a black-body radiation field with a physical temperature $T$,

$B_{v}(T)=\frac{2 h v^{3}}{c^{2}}\left[\mathrm{e}^{h v / k T}-1\right]^{-1}$,

the brightness temperature is thus

$\mathcal{J}_{v}(T)=\frac{\lambda^{2}}{2 k} B_{v}(T)=\frac{h v}{k}\left[\mathrm{e}^{h v / k T}-1\right]^{-1}$.

Note that expansion in $\frac{h v}{k T} \ll 1$ gives $\mathcal{J}_{v}(T) \approx T-\frac{h v}{2 k}\left[1-\frac{1}{6} \frac{h v}{k T}+O\left(\left(\frac{h v}{k T}\right)^{2}\right)\right]$. The difference between two black-bodies at temperatures $T_{2}$ and $T_{1}$ thus is

$\mathcal{J}_{v}\left(T_{2}\right)-\mathcal{J}_{v}\left(T_{1}\right) \approx\left(T_{2}-T_{1}\right)\left[1-\frac{1}{6}\left(\frac{h v}{k T_{1}}\right)\left(\frac{h v}{k T_{2}}\right)\right]=\left(T_{2}-T_{1}\right)[1-\delta]$.

With $\frac{h v}{k}=48 \mathrm{~K} \times v[\mathrm{THz}]$, the correction term for a frequency of $v=2 \mathrm{THz}$ and temperatures of $300 \mathrm{~K}$ and $77 \mathrm{~K}$ hence is $\delta=-0.066$, small but still significant, whereas at $345 \mathrm{GHz}$ it would only be $\delta=-0.0019$. obtain

Another quantity of interest is the ratio of black-body intensities between the image- and signal-sideband. With $v_{\mathrm{i}}=v_{\mathrm{s}} \pm v_{\mathrm{IF}}$, we

$\frac{\mathcal{J}_{v_{\mathrm{i}}}(T)}{\mathcal{J}_{v_{\mathrm{s}}}(T)} \approx 1 \mp\left(1+\frac{1}{3} \frac{h v}{k T}\right) \frac{h v_{\mathrm{IF}}}{k T}$

which, for $v=2 \mathrm{THz}, v_{\mathrm{IF}}=4 \mathrm{GHz}$ and a representative low temperature of $77 \mathrm{~K}$ gives an upper-/lower-sideband ratio of 1.0035 , i.e. within $0.35 \%$ of unity.

\section{A.2. Antenna temperatures}

We consider the general case of a heterodyne mixer that is sensitive in both sidebands and looks out to the sky, which emits an intensity equivalent to an antenna temperature $T_{\mathrm{A}, \mathrm{sky}, v}$, partially picks up intensity from ambient material with a physical temperature $T_{\mathrm{amb}}$, and possibly has a sideband filter that is terminated at a physical temperature of $T_{\text {term. }}$. The mixer then sees in a narrow frequency band, centered at a signal sideband frequency $v_{\mathrm{s}}$ and an image sideband frequency $v_{\mathrm{i}}$, a total intensity of

$$
\begin{aligned}
T_{\mathrm{A}}= & \eta_{\mathrm{mb}} t_{\mathrm{a}, v_{\mathrm{s}}} G_{\mathrm{s}} x_{\mathrm{s}} T_{\mathrm{MB}, v_{\mathrm{s}}}+\eta_{\mathrm{mb}} t_{\mathrm{a}, v_{\mathrm{i}}} G_{\mathrm{i}} x_{\mathrm{i}} T_{\mathrm{MB}, v_{\mathrm{i}}} \\
& +\left(1-f_{\mathrm{amb}}\right)\left[\left(1-t_{\mathrm{a}, v_{\mathrm{s}}}\right) G_{\mathrm{s}} x_{\mathrm{s}} \tilde{T}_{\mathrm{sky}, v_{\mathrm{s}}}+\left(1-t_{\mathrm{a}, v_{\mathrm{i}}}\right) G_{\mathrm{i}} x_{\mathrm{i}} \tilde{T}_{\mathrm{sky}, v_{\mathrm{i}}}\right] \\
& +f_{\mathrm{amb}}\left[G_{\mathrm{s}} x_{\mathrm{s}} \mathcal{J}_{v_{\mathrm{s}}}\left(T_{\mathrm{amb}}\right)+G_{\mathrm{i}} x_{\mathrm{i}} \mathcal{J}_{v_{\mathrm{i}}}\left(T_{\mathrm{amb}}\right)\right] \\
& +\left(1-x_{\mathrm{s}}\right) G_{\mathrm{s}} \mathcal{J}_{v_{\mathrm{s}}}\left(T_{\mathrm{term}}\right)+\left(1-x_{\mathrm{i}}\right) G_{\mathrm{i}} \mathcal{J}_{v_{\mathrm{i}}}\left(T_{\text {term }}\right),
\end{aligned}
$$

where we have introduced the following definitions:

$f_{\mathrm{amb}}$ : fraction of ambient material in sky beam;

$\eta_{\mathrm{mb}}:$ main beam efficiency;

$T_{\mathrm{mb}, \mathrm{s}, \mathrm{i}}$ : $\quad$ source main beam brightness temperature in signal(image) sideband;

$\tilde{T}_{\text {sky, },}$ : effective sky brightness temperature at frequency $v$,

$\tilde{T}_{\mathrm{sky}, v}:=T_{\mathrm{A}, \mathrm{sky}, v} /\left(1-t_{\mathrm{a}, v}\right)$;

$t_{\mathrm{a}, v}:$ atmospheric transmission at frequency $v$;

$G_{\mathrm{s}, \mathrm{i}}: \quad$ signal(image) sideband gain: $G_{\mathrm{s}}+G_{\mathrm{i}}=1$;

$x_{\mathrm{s}, \mathrm{i}}$ : $\quad$ signal(image) response in sky beam,

with coupling to sideband filter termination: $\left(1-x_{\mathrm{s}, \mathrm{i}}\right)$.

Here, we have assumed that the coupling factor to ambient and the main beam efficiency are independent of the reception sideband. 


\section{A.3. Types of receivers}

We can distinguish the following two cases straightforwardly:

- case A: a receiver with no sideband filter has

$x_{\mathrm{s}}=x_{\mathrm{i}}=1$

$\Rightarrow G_{\mathrm{s}} x_{\mathrm{s}}+G_{\mathrm{i}} x_{\mathrm{i}}=G_{\mathrm{s}}+G_{\mathrm{i}}=1 \Rightarrow G_{\mathrm{s}}=1-G_{\mathrm{i}}=\frac{1}{1+G_{\mathrm{i}} / G_{\mathrm{s}}}$.

GREAT on SOFIA falls into this category.

- case B: a receiver with a sideband filter, i.e., non-zero coupling to the sideband termination, has

$$
x_{\mathrm{s}}+x_{\mathrm{i}}=1 \Rightarrow x_{\mathrm{s}}=1-x_{\mathrm{i}}=\frac{1}{1+x_{\mathrm{i}} / x_{\mathrm{s}}} .
$$

Other hardware implementations correspond to different values of $G_{s, i}$ and $x_{s, i}$.

\section{A.4. Main-beam brightness temperature and forward beam brightness temperature}

In the above, we have expressed the source signal as a main-beam brightness temperature, i.e., as the convolution of the source brightness distribution on the sky with the main-beam profile of the telescope. The coupling of the detector to the telescope main beam is the main-beam efficiency $\eta_{\mathrm{mb}}$. This is appropriate if the telescope has a clean main beam, thus avoiding additional pick-up of source intensity in side-lobes (error-beam pickup). This is also a good description if the source is compact so that it has only intensity within the main beam.

Alternatively, the source signal can be expressed as a forward beam brightness temperature $T_{\mathrm{A}, v}^{*}$, i.e., as the convolution of the source brightness with the full forward antenna response. The relevant coupling efficiency is then the forward efficiency $\eta_{\mathrm{fwd}}$, which in the above nomenclature is $\eta_{\mathrm{fwd}}=1-f_{\mathrm{amb}}$. The term $\eta_{\mathrm{mb}} T_{\mathrm{MB}, v}$ then is replaced by $\eta_{\mathrm{fwd}} T_{\mathrm{A}, v}^{*}=\left(1-f_{\mathrm{amb}}\right) T_{\mathrm{A}, v}^{*}$.

\section{A.5. Sky brightness and opacity}

\section{A.5.1. Opacity}

The sky transmission along a line-of-sight at elevation $E l$ for a plane-parallel atmosphere, i.e., approximating the airmass by $A=$ $1 / \sin (E l)$ (the latter assumption being valid except at extremely low elevations), is

$t_{\mathrm{a}, \nu}=\mathrm{e}^{-\tau_{\nu} / \sin (E l)}$

is given by the zenith opacity $\tau_{v}$, where we drop the index $Z$ in the following.

The zenith-opacity of a particular species $\xi$ is the integral along the line-of-sight from the observers altitude $h$ to space over the abundance of that species times its absorption coefficient. The abundance at altitude $s$ is expressed as the volume-mixing ratio

$x_{\xi}(s)=n_{\xi}(s) / n(s)$

relative to the total density $n(s)$ (we closely follow the nomenclature of the AM handbook, Paine 2011). The general expression for the opacity of a particular atmospheric species $\xi$ with number density $n_{\xi}(s)=x_{\xi}(s) n(s)$ is then the line-of-sight integral over the product of its abundance times its pressure-, $P(s)$, and temperature-, $T(s)$, -dependent molecular absorption coefficient,

$\tau_{v, \xi}(h)=\int_{h}^{\infty} x_{\xi}(s) n(s) k_{\xi}(v, P(s), T(s)) \mathrm{d} s$.

The total opacity is obtained by summation over all species,

$\tau_{\nu}(h)=\sum_{\xi} \tau_{\nu, \xi}(h)$.

The details of the microphysics, i.e., the kind of absorption (single line transitions, CIA, etc.) are contained in the molecular absorption coefficients. For CIA the coefficient is proportional to the the abundance of the collision partner(s) $\hat{\xi}$, i.e.,

$k_{\xi}=x_{\hat{\xi}}(s) n(s) \kappa_{\xi, \hat{\xi}}^{C I A}(v, P(s), T(s))$,

which for self-broadening $(\xi=\hat{\xi})$ leads to a quadratic dependence on the abundance of the species.

Instead of altitude, the equivalent pressure can be used to specify the height in the atmosphere (pressure altitude) through the hydrostatic equation

$n(h) \mathrm{d} h=\frac{\mathrm{d} P}{m_{\text {air }} g}$,

with the mean molecular weight $m_{\text {air }}$ of the atmosphere and the gravitational acceleration $g$.

We will show below that the zenith opacity of the atmosphere above the observer can be separated into a wet and a dry part (where the dry part may contain opacity from the high water column in the stratosphere with constant volume mixing ratio that does not change with local humidity and daily weather). Thus, the opacity can be written as

$\tau_{v}(h)=b_{v}(h) p w v+c_{v}(h)$. 


\section{A.5.2. Sky brightness}

The sky brightness is the integral along the line-of-sight at elevation $E l$ over the source function times the attenuation, which for a plane-parallel layering, approximating the airmass as $A=1 / \sin (E l)$, reads

$T_{\mathrm{A}, \mathrm{sky}, v}=\int_{0}^{\tau_{v}} \mathcal{J}\left(T\left(\tau_{v}^{\prime}\right)\right) \exp \left(-\tau_{v}^{\prime} / \sin E l\right) \mathrm{d} \tau_{v}^{\prime} / \sin E l$.

From this, the effective sky brightness as defined in Eq. (A.7) can be calculated with a given line-of-sight transmission $t_{\mathrm{a}, v}$ as

$\tilde{T}_{\mathrm{sky}, v}=T_{\mathrm{A}, \mathrm{sky}, v} /\left(1-t_{\mathrm{a}, v}\right)$.

\section{A.6. Parameterization of the atmospheric water vapor}

For water, a reasonable altitude distribution in the stratosphere and upper troposphere is given by a low, constant mixing ratio, dominant in the upper stratosphere, plus an exponential term, extending through the upper troposphere and lower stratosphere (Mastenbrook 1968; Herbin et al. 2009). We thus write

$$
\begin{aligned}
x_{\mathrm{H}_{2} \mathrm{O}}(s) & =x_{\mathrm{c}}+x_{\mathrm{e}} \exp \left[-\left(s-h_{\mathrm{e}}\right) / h_{\mathrm{s}}\right] \\
& =x_{\mathrm{c}}+x_{\mathrm{e}}^{\prime} \exp \left(-s / h_{\mathrm{s}}\right),
\end{aligned}
$$

with $x_{\mathrm{e}}^{\prime}=x_{\mathrm{e}} \exp \left(h_{\mathrm{e}} / h_{\mathrm{s}}\right.$ ). The typical value for $x_{\mathrm{c}}$ is $2.5 \times 10^{-6}$ (Mastenbrook 1968; Herbin et al. 2009); $h_{\mathrm{c}}$ or $x_{\mathrm{e}}^{\prime}$ can be adjusted to match the desired column of water vapor above the observer. Equation (A.19) gives a total column density of water above altitude $h$ of

$$
\begin{aligned}
N_{\mathrm{H}_{2} \mathrm{O}}(h) & =\int_{h}^{\infty}\left(x_{\mathrm{c}}+x_{\mathrm{e}} \exp \left[-\left(s-h_{\mathrm{e}}\right) / h_{\mathrm{s}}\right]\right) n(s) \mathrm{d} s \\
& =x_{\mathrm{c}} \int_{h}^{\infty} n(s) \mathrm{d} s+x_{\mathrm{e}} \exp \left(h_{\mathrm{e}} / h_{\mathrm{s}}\right) \int_{h}^{\infty} \exp \left(-s / h_{\mathrm{s}}\right) n(s) \mathrm{d} s \\
& =x_{\mathrm{c}} N_{\mathrm{c}}(h)+x_{\mathrm{e}}^{\prime} N_{\mathrm{e}}(h),
\end{aligned}
$$

using the abbreviations

$$
\begin{aligned}
& N_{\mathrm{c}}(h)=\int_{h}^{\infty} n(s) \mathrm{d} s \\
& N_{\mathrm{e}}(h)=\int_{h}^{\infty} \mathrm{e}^{-s / h_{\mathrm{s}}} n(s) \mathrm{d} s .
\end{aligned}
$$

The minimum column is given by the constant volume mixing ratio, setting

$N_{\mathrm{H}_{2} \mathrm{O} \text {, min }}=x_{\mathrm{c}} N_{\mathrm{c}}(h)$.

Hence,

$x_{\mathrm{e}}^{\prime}=\left(N_{\mathrm{H}_{2} \mathrm{O}}(h)-x_{\mathrm{c}} N_{\mathrm{c}}(h)\right) / N_{\mathrm{e}}(h)$.

Similarly, using the abbreviations

$$
\begin{aligned}
K_{\mathrm{c}}(h) & =\int_{h}^{\infty} n(s) k_{\mathrm{H}_{2} \mathrm{O}}(v, P(s), T(s)) \mathrm{d} s \\
K_{\mathrm{e}}(h) & =\int_{h}^{\infty} n(s) \mathrm{e}^{-s / h_{\mathrm{s}}} k_{\mathrm{H}_{2} \mathrm{O}}(v, P(s), T(s)) \mathrm{d} s \\
K_{\mathrm{ee}}(h) & =\int_{h}^{\infty} n^{2}(s) \mathrm{e}^{-2 s / h_{\mathrm{s}}}, \kappa_{\mathrm{H}_{2} \mathrm{O}}^{\text {self }}(v, P(s), T(s)) \mathrm{d} s \\
K_{\mathrm{cc}}(h) & =\int_{h}^{\infty} n^{2}(s) \kappa_{\mathrm{H}_{2} \mathrm{O}}^{\text {self }}(v, P(s), T(s)) \mathrm{d} s \\
K_{\mathrm{ec}}(h) & =\int_{h}^{\infty} n^{2}(s) \mathrm{e}^{s / h_{\mathrm{s}}}, \kappa_{\mathrm{H}_{2} \mathrm{O}}^{\text {self }}(v, P(s), T(s)) \mathrm{d} s,
\end{aligned}
$$

the water opacity is

$$
\begin{aligned}
\tau_{v, \mathrm{H}_{2} \mathrm{O}}(h)= & x_{\mathrm{c}}\left\{K_{\mathrm{c}}(h)+x_{\mathrm{c}} K_{\mathrm{cc}}(h)\right\} \\
& +x_{\mathrm{e}}^{\prime}\left\{K_{\mathrm{e}}(h)+x_{\mathrm{e}}^{\prime} K_{\mathrm{ee}}(h)+x_{\mathrm{c}} K_{\mathrm{ec}}(h)\right\} \\
\approx & x_{\mathrm{c}} K_{\mathrm{c}}(h)+x_{\mathrm{e}}^{\prime} K_{\mathrm{e}}(h) .
\end{aligned}
$$


Note that in this notation, the absorption coefficient of water includes the contributions from CIA-processes due to collisions with other species,

$k_{\mathrm{H}_{2} \mathrm{O}}(v, P(s), T(s)) \rightarrow k_{\mathrm{H}_{2} \mathrm{O}}(v, P(s), T(s))+\sum_{\xi \neq \mathrm{H}_{2} \mathrm{O}} x_{\xi} n(s) \kappa_{\mathrm{H}_{2} \mathrm{O}, \xi}(v, P(S), T(s))$.

The self-broadening contribution for water in the thin, upper atmosphere can be neglected (Bauer et al. 1987, 1989). Combining the opacity contribution from the constant volume-mixing ratio part of water, $\tau_{\mathrm{c}, v}(h)=x_{\mathrm{c}} K_{\mathrm{c}}(h)$, with the opacity of all other species into

$c_{\nu}(h)=\tau_{\mathrm{c}, v}(h)+\sum_{\xi \neq \mathrm{H}_{2} \mathrm{O}} \tau_{v, \xi}(h)$

we can write the zenith opacity as separated into a dry opacity, resulting from all absorption from species other than water, but including the constant mixing ratio contribution from water in the upper stratosphere, and a wet opacity, which increases in proportion with the $p w v$ from the exponential part of the water vapor above the observer,

$p w v=\frac{\rho_{\mathrm{H}_{2} \mathrm{O}}}{m_{\mathrm{H}_{2} \mathrm{O}}}\left(N_{\mathrm{H}_{2} \mathrm{O}}(h)-N_{\mathrm{H}_{2} \mathrm{O}, \min }\right)$.

Hence, we obtain Eq. (A.16) with

$b_{v}(h)=\frac{m_{\mathrm{H}_{2} \mathrm{O}}}{\rho_{\mathrm{H}_{2} \mathrm{O}}} \frac{1}{N_{\mathrm{e}}(h)} K_{\mathrm{e}}(h)$.

\section{A.6.1. Alternative parameterization of the atmospheric water vapor}

Equation (A.19) is a rather ad hoc parameterization, which approximates the water abundance altitude profile above the SOFIA flight altitudes. An alternative parameterization, which is actually the one used in the present implementation with the kalibrate task in the kosma_software package, would be as follows:

$$
\begin{aligned}
x_{\mathrm{H}_{2} \mathrm{O}}(s) & = \begin{cases}x_{\mathrm{c}}, & h>h_{\mathrm{e}} \\
x_{\mathrm{c}} \mathrm{e}^{\left.-\left(s-h_{\mathrm{e}}\right) / h_{\mathrm{s}}\right)}, & h \leq h_{\mathrm{e}}\end{cases} \\
& =x_{\mathrm{c}} \mathrm{e}^{\left.-\left(s-h_{\mathrm{e}}\right) / h_{\mathrm{s}}\right)}+x_{\mathrm{c}} \begin{cases}1-\mathrm{e}^{\left.-\left(s-h_{\mathrm{e}}\right) / h_{\mathrm{s}}\right)}, & h>h_{\mathrm{e}} \\
0, & h \leq h_{\mathrm{e}}\end{cases}
\end{aligned}
$$

This gives, using $N_{\mathrm{e}}(h)$ and $N_{\mathrm{c}}(h)$ as defined in Eq. (A.21) above

$$
\begin{aligned}
N_{\mathrm{H}_{2} \mathrm{O}}(h) & =x_{\mathrm{c}} \mathrm{e}^{h_{\mathrm{e}} / h_{\mathrm{s}}}\left\{\int_{h}^{\infty} \mathrm{e}^{-\frac{s}{h_{\mathrm{s}}}} n(s) \mathrm{d} s+\mathrm{e}^{-\frac{h_{\mathrm{e}}}{h_{\mathrm{s}}}} \int_{h_{\mathrm{e}}}^{\infty}\left(1-\mathrm{e}^{-\frac{s-h_{\mathrm{e}}}{h_{\mathrm{s}}}}\right) n(s) \mathrm{d} s\right\} \\
& =x_{\mathrm{c}} \mathrm{e}^{h_{\mathrm{e}} / h_{\mathrm{s}}}\left\{N_{\mathrm{e}}(h)-N_{\mathrm{e}}\left(h_{\mathrm{e}}\right)+\mathrm{e}^{-h_{\mathrm{e}} / h_{\mathrm{s}}} N_{\mathrm{c}}\left(h_{\mathrm{e}}\right)\right\} .
\end{aligned}
$$

As in the case above, $h_{\mathrm{e}}$ can be varied to scale the water profile to the desired total column of water above the observer. Owing to the different parameterization, the variation of $N_{\mathrm{H}_{2} \mathrm{O}}(h)$ with $h_{\mathrm{e}}$ is now a more complex dependence and solving for $h_{\mathrm{e}}$ is not possible analytically. The minimum column of water above the observer is now given by the contribution with the constant volume mixing ratio when $h_{\mathrm{e}}=h$, namely

$$
\left.N_{\mathrm{H}_{2} \mathrm{O}}\right|_{h_{\mathrm{e}}=h}=x_{\mathrm{c}} \int_{h}^{\infty} n(s) \mathrm{d} s=x_{\mathrm{c}} N_{\mathrm{c}}(h),
$$

identical to the case above. Similarly, and again neglecting the self-broadening contributions of water, the water opacity is now given by

$\tau_{v, \mathrm{H}_{2} \mathrm{O}}=x_{\mathrm{c}} \mathrm{e}^{h_{\mathrm{e}} / h_{\mathrm{s}}}\left\{K_{\mathrm{e}}(h)-K_{\mathrm{e}}\left(h_{\mathrm{e}}\right)+\mathrm{e}^{-h_{\mathrm{e}} / h_{\mathrm{s}}} K_{\mathrm{c}}(h)\right\}$.

For small increases above the minimum, i.e. $h_{\mathrm{e}}=h+\epsilon$, we have

$$
\begin{aligned}
\left.N_{\mathrm{H}_{2} \mathrm{O}}\right|_{h_{\mathrm{e}}=h} ^{h_{\mathrm{e}}=h+\epsilon}= & x_{\mathrm{c}} \mathrm{e}^{(h+\epsilon) / h_{\mathrm{s}}}\left(N_{\mathrm{e}}(h)-N_{\mathrm{e}}(h+\epsilon)\right) \\
& +x_{\mathrm{c}}\left(N_{\mathrm{c}}\left(h_{\mathrm{e}}\right)-N_{\mathrm{c}}(h)\right) \\
= & x_{\mathrm{c}} \mathrm{e}^{(h+\epsilon) / h_{\mathrm{s}}} \int_{h}^{h+\epsilon} n(s) \mathrm{d} s-x_{\mathrm{c}} \int_{h}^{h+\epsilon} n(s) \mathrm{d} s \\
\stackrel{\epsilon \rightarrow 0}{\rightarrow} & x_{\mathrm{c}} \mathrm{e}^{h / h_{\mathrm{s}}}\left(1+\frac{\epsilon}{h_{\mathrm{s}}}\right) n(h) \epsilon-x_{\mathrm{c}} n(h) \epsilon \\
= & x_{\mathrm{c}} \frac{n(h)}{h_{\mathrm{s}}} \epsilon^{2} .
\end{aligned}
$$


For the water opacity we obtain, following Eq. (A.30), and abbreviating $k(s)=k_{\mathrm{H}_{2} \mathrm{O}}(v, P(s), T(s))$,

$$
\begin{aligned}
\left.\tau_{v, \mathrm{H}_{2} \mathrm{O}}\right|_{h_{\mathrm{e}}=h} ^{h_{\mathrm{e}}=h+\epsilon}= & x_{\mathrm{c}} \mathrm{e}^{(h+\epsilon) / h_{\mathrm{s}}}\left(K_{\mathrm{e}}(h)-K_{\mathrm{e}}(h+\epsilon)\right) \\
& +K_{\mathrm{c}}\left(N_{\mathrm{c}}\left(h_{\mathrm{e}}\right)-K_{\mathrm{c}}(h)\right) \\
= & x_{\mathrm{c}} \mathrm{e}^{(h+\epsilon) / h_{\mathrm{s}}} \int_{h}^{h+\epsilon} n(s) k(s) \mathrm{d} s-x_{\mathrm{c}} \int_{h}^{h+\epsilon} n(s) k(s) \mathrm{d} s \\
\stackrel{\epsilon \rightarrow 0}{\rightarrow} & x_{\mathrm{c}} \mathrm{e}^{h / h_{\mathrm{s}}}\left(1+\frac{\epsilon}{h_{\mathrm{s}}}\right) n(h) k(h) \epsilon-x_{\mathrm{c}} n(h) k(h) \epsilon \\
= & x_{\mathrm{c}} \frac{n(h)}{h_{\mathrm{s}}} k(h) \epsilon^{2},
\end{aligned}
$$

so that the opacity is proportional to pwv, with

$b_{v}(h)=\frac{\rho_{\mathrm{H}_{2} \mathrm{O}}}{m_{\mathrm{H}_{2} \mathrm{O}}} k_{\mathrm{H}_{2} \mathrm{O}}(v, P(h), T(h))$.

For larger water columns, the opacity is not necessarily proportional to $p w v$ : although the $N_{\mathrm{e}^{-}}$, resp. $K_{\mathrm{c}^{-}}$, contribution in Eqs. (A.34) and (A.35) becomes rapidly insignificant relative to the first terms because of the exponential decrease with increasing $h_{\mathrm{e}}$ : the variation of the altitude distribution due to the changing point of intersection between the constant and exponential part, $h_{\mathrm{e}}$, results in changing line profiles in the first terms. However, as long as the variation of the line profile with altitude is relatively slow, i.e. $k_{v}(s) \approx k_{v}(h)$, we approximately have

$K_{\mathrm{e}}(h)-K_{\mathrm{e}}\left(h_{\mathrm{e}}\right) \approx k_{v}(h)\left(N_{\mathrm{e}}(h)-N_{\mathrm{e}}\left(h_{\mathrm{e}}\right)\right)$,

so that the proportionality between opacity and water column holds again, with Eq. (A.36) holding approximately.

\section{A.7. Spectrometer response and gain profile}

The spectrometer detects in each frequency channel (we omit the index specifying the spectrometer channel, resp. intermediate frequency, $v_{\mathrm{IF}}$, in the following) a count rate (counts/sec) $C$ proportional to the radiation input plus the receiver noise (expressed as an equivalent brightness temperature at the receiver input, $T_{\text {rec }}$ ), the total being

$T_{\mathrm{sys}}=T_{\mathrm{rec}}+T_{\mathrm{A}}$.

Additionally, depending on the detailed detection mechanism of the power in the spectrometer channels, the receiver may have a count-offset $Z$, i.e., a non-zero count rate at zero (IF-) input power. The detected count rate is thus

$C=\gamma\left(T_{\mathrm{A}}+T_{\mathrm{rec}}\right)+Z$

so that the spectrometer gain $\gamma$ (gain profile, if specified as a function of frequency) determines the response of the receiver for any difference measurement:

$\Delta C=C_{2}-C_{1}=\gamma\left(T_{\mathrm{A}, 2}-T_{\mathrm{A}, 1}\right)=\gamma \Delta T_{\mathrm{A}}$.

\section{A.8. Gain calibration: Hot- and cold-load measurements}

For calibration measurements on the hot- or cold-load, the mixer sees

$$
\begin{aligned}
T_{\mathrm{A} \text {, hold }}^{\text {hot }}= & \left(G_{\mathrm{s}} x_{\mathrm{s}}+G_{\mathrm{i}} x_{\mathrm{i}}\right) T_{\text {hot }}^{\prime} \\
& +\left(1-x_{\mathrm{s}}\right) G_{\mathrm{s}} \mathcal{J}_{v_{\mathrm{s}}}\left(T_{\text {term }}\right)+\left(1-x_{\mathrm{i}}\right) G_{\mathrm{i}} \mathcal{J}_{v_{\mathrm{s}}}\left(T_{\text {term }}\right),
\end{aligned}
$$

where we have used the abbreviation

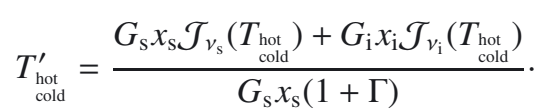

With the known physical temperatures of the loads, and the known sideband gains and sideband filter coupling coefficients, the spectrometer gain in each spectral resolution element can be measured from the difference in count rates between the hot- and cold-load measurement:

$\gamma=\frac{C_{\mathrm{hot}}-C_{\text {cold }}}{T_{\mathrm{A}, \mathrm{hot}}-T_{\mathrm{A}, \text { cold }}}=\frac{C_{\mathrm{hot}}-c_{\mathrm{cold}}}{\left(G_{\mathrm{s}} x_{\mathrm{s}}+G_{\mathrm{i}} x_{\mathrm{i}}\right)\left(\left(T_{\mathrm{hot}}^{\prime}-T_{\text {cold }}^{\prime}\right)\right.}$.

As discussed above, the difference between signal- and image-sideband brightness temperatures may be neglected to relative high precision, resulting in $T_{\text {hot }}^{\prime}=\mathcal{J}_{v}\left(T_{\text {hot }}\right)$, where an obviously consistent choice for the frequency at which to evaluate the brightnesses would be $v=\frac{1}{2}\left(v_{\mathrm{s}}+v_{\mathrm{i}}\right)=v_{\mathrm{LO}}$.

L4, page 10 of 13 


\section{A.9. Sky transmission calibration}

The sky transmission is derived from measurements on the blank sky (either explicit sky-measurements, or the off-source positions of an astronomical observation). The measured difference between sky- and hot-load measurement gives

$$
\left.\left(T_{\mathrm{A}, \mathrm{sky}}-T_{\mathrm{A}, \mathrm{hot}}\right)\right|_{\text {meas }}=\frac{C_{\text {sky }}-C_{\text {hot }}}{\gamma}
$$

(note that here and in the following, the cold-load measurement and temperatures may be used instead of the hot-load). This is compared with the theoretical expression for the brightness difference, which, using Eqs. (A.6) and (A.41), gives

$$
\begin{aligned}
\left.\left(T_{A . \mathrm{sky}}-T_{\mathrm{A}, \mathrm{hot}}\right)\right|_{\text {meas }}= & \left(1-f_{\mathrm{amb}}\right) \\
& \times\left[\left(1-t_{\mathrm{a}, v_{\mathrm{s}}}\right) G_{\mathrm{s}} x_{\mathrm{s}} \tilde{T}_{\mathrm{sky}, v_{\mathrm{s}}}+\left(1-t_{\mathrm{a}, v_{\mathrm{i}}}\right) G_{\mathrm{i}} x_{\mathrm{i}} \tilde{T}_{\mathrm{sky}, v_{\mathrm{i}}}\right] \\
& +f_{\mathrm{amb}}\left[G_{\mathrm{s}} x_{\mathrm{s}} \mathcal{J}_{v_{\mathrm{s}}}\left(T_{\mathrm{amb}}\right)+G_{\mathrm{i}} x_{\mathrm{i}} \mathcal{J}_{v_{\mathrm{i}}}\left(T_{\mathrm{amb}}\right)\right] \\
& -\left[G_{\mathrm{s}} x_{\mathrm{s}} \mathcal{J}_{v_{\mathrm{s}}}\left(T_{\mathrm{hot}}\right)+G_{\mathrm{i}} x_{\mathrm{i}} \mathcal{J}_{v_{\mathrm{i}}}\left(T_{\mathrm{hot}}\right)\right] \\
= & \left(1-f_{\mathrm{amb}}\right)\left\{G_{\mathrm{s}} x_{\mathrm{s}}\left[\tilde{T}_{\mathrm{sky}, v_{\mathrm{s}}}\left(1-t_{\mathrm{a}, v_{\mathrm{s}}}\right)-\tilde{T}_{\mathrm{hot}, v_{\mathrm{s}}}\right]\right. \\
& \left.\quad+G_{\mathrm{i}} x_{\mathrm{i}}\left[\tilde{T}_{\mathrm{sky}, v_{\mathrm{i}}}\left(1-t_{\mathrm{a}, v_{\mathrm{i}}}\right)-\tilde{T}_{\mathrm{hot}, v_{\mathrm{i}}}\right]\right\}
\end{aligned}
$$

where we have used the abbreviation for the effective hot-temperature

$\tilde{T}_{\mathrm{hot}, v}=\frac{\mathcal{J}_{v}\left(T_{\mathrm{hot}}\right)-f_{\mathrm{amb}} \mathcal{J}_{v}\left(T_{\mathrm{amb}}\right)}{1-f_{\mathrm{amb}}}$.

Introducing the additional approximations

$a_{\mathrm{s}}=\frac{\tilde{T}_{\mathrm{sky}, v_{\mathrm{s}}}}{\tilde{T}_{\mathrm{hot}, v_{\mathrm{s}}}}, a_{i}=\frac{\tilde{T}_{\mathrm{sky}, v_{\mathrm{i}}}}{\tilde{T}_{\mathrm{hot}, \nu_{\mathrm{i}}}}$,

$\Gamma=\frac{G_{\mathrm{i}} x_{\mathrm{i}}}{G_{\mathrm{s}} x_{\mathrm{s}}}$

$\omega=\frac{\tilde{T}_{\text {sky, } v_{\mathrm{i}}}}{\tilde{T}_{\mathrm{sky}, v_{\mathrm{s}}}}$

$\Omega=\frac{\tilde{T}_{\text {hot }, v_{\mathrm{i}}}-\tilde{T}_{\mathrm{sky}, v_{\mathrm{i}}}}{\tilde{T}_{\text {hot }, v_{\mathrm{s}}}-\tilde{T}_{\mathrm{sky}, v_{\mathrm{s}}}}$

gives

$$
\begin{aligned}
\left.\left(T_{\mathrm{A}, \mathrm{sky}}-T_{\mathrm{A}, \mathrm{hot}}\right)\right|_{\text {meas }}= & -\left(1-f_{\mathrm{amb}}\right) \tilde{T}_{\mathrm{hot}, v_{\mathrm{s}}} a_{\mathrm{s}} G_{\mathrm{s}} x_{\mathrm{s}}(1+\Gamma) \\
& \times\{t_{\mathrm{a}, v_{\mathrm{s}}} \underbrace{\frac{1+\Gamma \omega\left(t_{\mathrm{a}, v_{\mathrm{i}}} / t_{\mathrm{a}, v_{\mathrm{s}}}\right)}{1+\Gamma}}_{1 / \alpha}+\frac{1-a_{\mathrm{s}}}{a_{\mathrm{s}}} \frac{1+\Gamma \Omega}{1+\Gamma}\} \\
= & -\left(1-f_{\mathrm{amb}}\right) \tilde{T}_{\mathrm{sky}, v_{\mathrm{s}}} G_{\mathrm{s}} x_{\mathrm{s}} \\
& \times\left\{t_{\mathrm{a}, v_{\mathrm{s}}}\left(1+\Gamma \omega\left(t_{\mathrm{a}, v_{\mathrm{i}}} / t_{\mathrm{a}, v_{\mathrm{s}}}\right)\right)+\frac{1-a_{\mathrm{s}}}{a_{\mathrm{s}}}(1+\Gamma \Omega)\right\} .
\end{aligned}
$$

As discussed above, $\omega \approx 1$ to high precision for the relevant range of frequencies and temperatures. $\Omega \approx 1$ only holds as long as the effective sky brightness is close to a black-body function, i.e., $\tilde{T}_{\text {sky }, v} \approx \mathcal{J}_{v}\left(T_{\text {sky }}\right)$, with a frequency-independent effective physical sky temperature $T_{\text {sky }}$. This is not necessarily the case: a narrow-line absorption in the signal band resulting from water at the low temperatures of the upper troposphere and a similarly narrow feature from a high-altitude dry species at the higher physical temperature of the upper stratosphere may have very different effective sky temperatures, following their definitions according to Eqs. (A.17) and (A.18).

Assuming for simplicity $\Omega=1$ and $\omega=1$, and considering a DSB receiver with equal gain in both sidebands and no sideband filter, i.e., $\Gamma=1, x_{\mathrm{s}}=1$ and $G_{\mathrm{s}}=1$, we obtain the simple formula

$$
\left.\left(T_{\mathrm{A}, \mathrm{sky}}-T_{\mathrm{A}, \mathrm{hot}}\right)\right|_{\text {meas }}=-\left(1-f_{\mathrm{amb}}\right) \mathcal{J}_{v_{\mathrm{s}}}\left(T_{\mathrm{sky}}\right)\left\{\frac{1}{2}\left(t_{\mathrm{a}, v_{\mathrm{s}}}+t_{\mathrm{a}, v_{\mathrm{i}}}\right)+\frac{1-a_{\mathrm{s}}}{a_{\mathrm{s}}}\right\} \text {. }
$$


For a single-sideband receiver, i.e., $G_{\mathrm{i}}=0$, and hence $\Gamma=0, x_{\mathrm{s}}=1$ and $G_{\mathrm{s}}=1$, we obtain

$\left.\left(T_{\mathrm{A}, \mathrm{sky}}-T_{\mathrm{A}, \mathrm{hot}}\right)\right|_{\text {meas }}=-\left(1-f_{\mathrm{amb}}\right) \mathcal{J}_{v_{\mathrm{s}}}\left(T_{\mathrm{sky}}\right)\left\{t_{\mathrm{a}, v_{\mathrm{s}}}+\frac{1-a_{\mathrm{s}}}{a_{\mathrm{s}}}\right\}$.

The latter can be immediately inverted to calculate the sky transmission in the signal band; in the former case, only the average transmission in the signal- and image-sideband can be determined.

The same holds for the general case of Eq. (A.48), which gives

$t_{\mathrm{a}, v_{\mathrm{s}}}+\Gamma \omega t_{\mathrm{a}, v_{\mathrm{i}}}=\frac{\left(C_{\mathrm{hot}}-C_{\mathrm{sky}}\right) / \gamma}{\left(1-f_{\mathrm{amb}}\right) \tilde{T}_{\mathrm{sky}, v_{\mathrm{s}}} G_{\mathrm{s}} x_{\mathrm{s}}}-\frac{1-a_{\mathrm{s}}}{a_{\mathrm{s}}}(1+\Gamma \Omega)$,

from which the sky transmission in the signal-sideband alone can be determined only with additional knowledge on i) the ratio of signal- and image-sideband transmission, and ii) the effective sky brightness at the signal- and image-band frequency, $\tilde{T}_{\text {sky, } v_{s, i}}$, which is also needed to calculated $\Omega$ with full precision.

Determining the transmission across the spectrometer channel by channel is not the best strategy, because the values derived would be very noisy due to the relatively low $\mathrm{S} / \mathrm{N}$ per spectrometer channel. This can be improved by using broader frequency averages, but at the cost of loosing the information of variations of the atmospheric absorption with frequency on frequency scales below the averaging interval.

To overcome the difficulty of the unknown atmospheric transmission ratio between image- and signal-sideband, one has to use additional knowledge about the atmosphere by applying an atmospheric model and fitting the observed sky-hot brightness to the model-predicted one.

This is implemented in the KOSMA-observing software package kosma_software in the task kalibrate as follows: (i) the first step is to identify and discard "bad" channels, e.g., at the band edges without significant IF-response and saturated channels etc.; (ii) the observed and calibrated sky-hot difference across the reception bandwidth of the receiver is then fitted with the atmospheric model prediction, following Eq. (A.48), using the $p w v$ as a free parameter; the implementation at present makes the simplifying assumption of $\omega=1$ and $\Omega=1$. The best-fit value is thus determined with high precision, making use of the full spectral information observed, i.e., with a good S/N. The user can control whether the fit is performed as a "common" fit to all simultaneously observed spectra (several receiver pixels at several frequency bands) or individually for each spectrometer; (iii) the measured on-off source spectrum can then be calibrated on the main-beam brightness temperature scale via

$\Delta T_{\mathrm{mb}, \mathrm{SSB}}=\frac{C_{\mathrm{ON}}-C_{\mathrm{OFF}}}{\eta_{\mathrm{mb}} \gamma G_{\mathrm{s}} x_{\mathrm{s}}} \exp \left(b_{v} p w v+c_{v}\right)$

This is made separately for each spectrometer channel, thus applying the frequency dependence of the atmospheric transmission as specified by the atmospheric model to the data.

\section{A.10. Determination of receiver noise and system temperature}

Equations (A.38) and (A.39) define the system temperature used for proper noise-weighting of the data when averaging, and the receiver temperature that needs to be optimized when tuning the receiver, both on a $T_{\mathrm{A}}$ scale, however. Comparison with Eq. (A.41) shows that the system temperature and receiver temperature referred to an equivalent black-body with physical temperature $T_{\text {sys,phys, }}$, resp. $T_{\text {rec,phys }}$ at the receiver input, either DSB or single-sideband, is

$T_{\text {rec,phys,DSB }}=\frac{T_{\text {rec }}}{G_{\mathrm{s}} x_{\mathrm{S}}+G_{\mathrm{i}} x_{\mathrm{i}}} \quad T_{\text {rec,phys, } \mathrm{SSB}}=\frac{T_{\text {rec }}}{G_{\mathrm{s}} x_{\mathrm{s}}}$

respectively

$T_{\text {sys,phys,DSB }}=\frac{T_{\text {sys }}}{G_{\mathrm{s}} x_{\mathrm{s}}+G_{\mathrm{i}} x_{\mathrm{i}}} \quad T_{\text {sys,phys, } \mathrm{SSB}}=\frac{T_{\mathrm{sys}}}{G_{\mathrm{s}} x_{\mathrm{s}}}$.

From the defining Eq. (A.39), we obtain for the hot- and cold-load measurement

$C_{\text {hot }}-z=\gamma\left(T_{\text {rec }}+T_{\mathrm{A}, \mathrm{hot}}\right)$

$C_{\text {cold }}-z=\gamma\left(T_{\text {rec }}+T_{\text {A,cold }}\right)$,

and can derive ( $Y$-factor method)

$Y=\frac{C_{\text {hot }}-z}{C_{\text {cold }}-z}=\frac{T_{\text {rec }}+T_{\mathrm{A}, \text { hot }}}{T_{\text {rec }}+T_{\mathrm{A}, \text { cold }}}$.

Solving for $T_{\text {rec }}$ gives

$$
\begin{aligned}
T_{\text {rec }}= & \frac{T_{\mathrm{A}, \text { hot }}-Y T_{\mathrm{A}, \text { cold }}}{Y-1} \\
= & \underbrace{\frac{T_{\text {hot }}^{\prime}-Y T_{\text {cold }}^{\prime}}{Y-1}\left(G_{\mathrm{s}} x_{\mathrm{s}}+G_{\mathrm{i}} x_{\mathrm{i}}\right)}_{T_{\text {rec }}^{\prime}} \\
& -\left[G_{\mathrm{s}}\left(1-x_{\mathrm{s}}\right) \mathcal{J}_{v_{\mathrm{s}}}\left(T_{\text {term }}\right)+G_{\mathrm{i}}\left(1-x_{\mathrm{i}}\right) \mathcal{J}_{v_{\mathrm{i}}}\left(T_{\text {term }}\right)\right] \\
= & T_{\text {rec }}^{\prime}\left(G_{\mathrm{s}} x_{\mathrm{s}}+G_{\mathrm{i}} x_{\mathrm{i}}\right)-\left[G_{\mathrm{s}}\left(1-x_{\mathrm{s}}\right) \mathcal{J}_{v_{\mathrm{s}}}\left(T_{\text {term }}\right)+G_{\mathrm{i}}\left(1-x_{\mathrm{i}}\right) \mathcal{J}_{v_{\mathrm{i}}}\left(T_{\text {term }}\right)\right],
\end{aligned}
$$

L4, page 12 of 13 
so that

$$
\begin{aligned}
& T_{\text {rec,phys,DSB }}=T_{\text {rec }}^{\prime}-\frac{G_{\mathrm{s}}\left(1-x_{\mathrm{s}}\right) \mathcal{J}_{v_{\mathrm{s}}}\left(T_{\text {term }}\right)+G_{\mathrm{i}}\left(1-x_{\mathrm{i}}\right) \mathcal{J}_{\mathrm{v}_{\mathrm{i}}}\left(T_{\text {term }}\right)}{G_{\mathrm{s}} x_{\mathrm{s}}+G_{\mathrm{i}} x_{\mathrm{i}}} \\
& T_{\text {rec,phys,SSB }}=T_{\text {rec }}^{\prime}(1+\Gamma)-\frac{G_{\mathrm{s}}\left(1-x_{\mathrm{s}}\right) \mathcal{J}_{v_{\mathrm{s}}}\left(T_{\text {term }}\right)+G_{\mathrm{i}}\left(1-x_{\mathrm{i}}\right) \mathcal{J}_{v_{\mathrm{i}}}\left(T_{\text {term }}\right)}{G_{\mathrm{s}} x_{\mathrm{s}}} .
\end{aligned}
$$

Ignoring the difference in brightness between the signal- and image-sideband on the hot- and cold load, $T_{\text {rec }}^{\prime}$ reduces to the standard formula for the receiver temperature in the $Y$-factor method,

$T_{\text {rec }}^{\prime} \approx \frac{\mathcal{J}_{v}\left(T_{\text {hot }}\right)-Y \mathcal{J}_{v}\left(T_{\text {cold }}\right)}{Y-1}$

The additional term on the right-hand-side corrects for the coupling to the image-sideband termination in the sideband filter and reduces to 0 with $x_{\mathrm{s}}=x_{\mathrm{i}}=1$.

We obtain for the system temperature (on the off-source position on the sky)

$$
\begin{aligned}
T_{\text {sys }} & =T_{\text {rec }}+T_{\mathrm{A}, \text { sky }} \\
& =\left(T_{\text {rec }}+T_{\mathrm{A}, \mathrm{hot}}\right)+\left(T_{\mathrm{A}, \mathrm{sky}}-T_{\mathrm{A}, \mathrm{hot}}\right) \\
& =\left(T_{\mathrm{rec}}^{\prime}+T_{\mathrm{hot}}^{\prime}\right) G_{\mathrm{s}} x_{\mathrm{s}}(1+\Gamma)+\left(T_{\mathrm{A}, \mathrm{sky}}-T_{\mathrm{A}, \mathrm{hot}}\right) .
\end{aligned}
$$

Insertingly, we obtain from Eq. (A.48)

$$
\begin{aligned}
T_{\mathrm{sys}}= & G_{\mathrm{s}} x_{\mathrm{s}}(1+\Gamma) \\
& \times\left[T_{\mathrm{rec}}^{\prime}+T_{\mathrm{hot}}^{\prime}-\tilde{T}_{\mathrm{sky}, v_{\mathrm{s}}}\left(1-f_{\mathrm{amb}}\right)\left(t_{\mathrm{a}, v_{\mathrm{s}}} \frac{1}{\alpha}+\frac{1-a_{\mathrm{s}}}{a_{\mathrm{s}}} \frac{1+\Gamma \Omega}{1+\Gamma}\right)\right],
\end{aligned}
$$

and $T_{\text {sys,DSB }}$ or $T_{\text {sys,SSB }}$ following Eq. (A.54). 